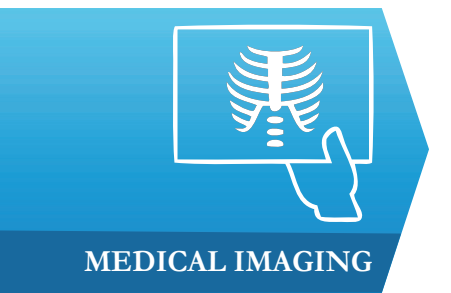

\section{Cardiovascular magnetic resonance: contribution to the exploration of cardiomyopathies}

\author{
Călin Schiau ${ }^{1}$, Șerban Schiau ${ }^{2}$, Sorin M. Dudea ${ }^{1,3}$, Simona Manole $^{1}$
}

1) Radiology Department, Iuliu Hatieganu University of Medicine and Pharmacy, Cluj-Napoca, Romania

2) "Niculae Stancioiu" Heart Institute, Cluj-Napoca, Romania

3) Radiology Department, Emergency Clinical County Hospital, Cluj-Napoca, Romania
DOI: $10.15386 / \mathrm{mpr}-1343$

Manuscript received: 15.04.2019

Received in revised form: 14.07.2019

Accepted: 31.07 .2019

Address for correspondence:

calin.schiau@yahoo.com

\begin{abstract}
Background and aims. Magnetic resonance imaging is a non-invasive and nonirradiating imaging method, complementary to cardiac ultrasound in the assessment of cardiovascular disease and implicitly of cardiomyopathies. Although it is not a first intention imaging method, it is superior in the assessment of cardiac volumes, left ventricular ejection fraction, in the analysis of cardiac wall dyskinesia and myocardial tissue characteristics with and without using a contrast agent. The purpose of this paper is to review the current knowledge regarding cardiovascular magnetic resonance imaging (CMR) and its applications in cardiomyopathy analysis.
\end{abstract}

Methods. In order to create this review, relevant articles were searched and analyzed by using MeSH terms such as: "cardiac magnetic resonance imaging", "cardiomyopathy", "myocardial fibrosis". Three main international databases PubMed, Web of Science and Medscape were searched. We carried out a narrative review focused on the current indications of cardiovascular magnetic resonance imaging in cardiomyopathies, both common and raret, of ischemic and nonischemic types.

Results. Cardiac magnetic resonance imaging has a very important role in the diagnosis, assessment and prognosis of common cardiomyopathies (the dilated, hypertrophic and inflammatory types) or other more rare ones such as (amyloidosis, arrhythmogenic right ventricular, non-compaction or Takotsubo cardiomyopathy), as it represents the gold standard for evaluating the ejection fraction, ventricular volumes and mass. CMR techniques, such as late gadolinium enhancement, T1 and T2 mapping have proven their usefulness, helping differentiate between ischemic (subendocardial enhancement) and nonischemic cardiomyopathy (varied pattern) or also establish the etiology. Another important feature of this imaging technique is that it can establish the myocardial viability, thus the chance of contractile recovery after revascularization. This feature is based on the transmural extent of LGE, left ventricle wall thickness and the assessment of the contractile reserve after administration of low dose dobutamine.

Conclusions. Cardiovascular magnetic resonance imaging is an indispensable tool, with proven efficiency, capable of providing the differential diagnosis between ischemic and nonischemic cardiomyopathy or establishing the etiology in the nonischemic type. In addition, these findings have a prognostic value, they may guide the patient management plan and, if necessary, can evaluate treatment response. Therefore, this technique should be part of any routine investigation of various cardiomyopathies.

Keywords: cardiovascular magnetic resonance imaging, late gadolinium enhancement, myocardial fibrosis, cardiomyopathy, ischemic and nonischemic cardiomyopathy

\section{Introduction}

Cardiomyopathies are defined as a heterogeneous group of myocardial diseases associated with mechanical and / or electrical dysfunction, which may be accompanied by hypertrophy or dilation, having various causes, often genetic, in the absence of hypertension, valvular disease, coronary artery disease and congenital heart disease. The myocardial damage may also be a manifestation of systemic disorder [1].

Cardiomyopathies can be classified into 2 groups according to the American Heart Association (AHA), namely: primary (genetic / mixed / acquired) and secondary. 
The European Society of Cardiology (ESC) classifies the cardiomyopathies taking into account the pathophysiological mechanism. According to ESC, they are divided into: dilated cardiomyopathy (DCM), hypertrophic cardiomyopathy (HCM), restrictive cardiomyopathy (RCM), cardiomyopathy/dysplasia (ARVC/D), specific cardiomyopathies and non-classifiable CM [1]. The specific CM may be secondary to ischemia, hypertension, congenital heart disease, arrhythmogenic right ventricular (RV), valvulopathy and chronic pulmonary disease.

Cardiomyopathies are more common than expected, affecting up to $50 \%$ of patients with sudden cardiac death in childhood or adolescence and a significant number of the candidates for a heart transplant [2].

The most frequently encountered cardiomyopathies are dilated cardiomyopathy (DCM), hypertrophic cardiomyopathy (HCM), restrictive cardiomyopathy (RCM) and arrhythmogenic right ventricular cardiomyopathy (ARVC) [3]. The most common is DCM, affecting 5/100.000 adults and 0.57/100.000 children [4,5]. Another common CM is HCM, an important cause of sudden cardiac death (SCD) in athletes, 1/500 of them being affected [3]. Restrictive and arrhythmogenic right ventricular $\mathrm{CM}$ are more rare [6].

In order to create this review, relevant articles were searched and analyzed by using MeSH terms such as: "cardiac magnetic resonance imaging", "cardiomyopathy", "myocardial fibrosis". Three main international databases PubMed, Web of Science and Medscape were searched. A number of 1133 relevant articles were found. Articles that contained information about MRI assessment of cardiomyopathies, their etiology or complications after coronary reperfusion therapy were included. Articles about cardiomyopathies, but explored otherwise than by magnetic resonance imaging, case presentations, or written in languages with limited circulation were excluded.

From the remaining articles, only 66 are cited in this review, as the most current, relevant and essential for our research.

The aim of this paper is to summarize and highlight the important role of cardiovascular magnetic resonance imaging (CMR) in the diagnosis, treatment guidance and prognosis of cardiomyopathies.

\section{Modern imaging techniques in cardiomyopathies}

Cardiac imaging has become a very important tool of any routine cardiac investigation as it is capable of detecting early disease manifestations, and is able to provide accurate and objective quantification of cardiovascular abnormalities.

The modern advanced imaging techniques such as echocardiography and magnetic resonance imaging are able to provide information about heart function and structure, obtaining data about the size of cardiac chambers, wall thickness and motion abnormalities, left ventricular ejection fraction (LVEF), diastolic function, stroke volume (SV) and cardiac output $(\mathrm{CO})$, pressure estimations, detection of myocardial fibrosis or infarcted tissue [7].

Nowadays, a routine investigation includes physical examination, clinical history, laboratory testing, but these alone are often insufficient for a proper diagnosis; so, cardiac imaging has gradually gained an important role, becoming today, in most cases, indispensable, especially due to its non-invasiveness compared to the gold standard for myocardial tissue analysis of CM - endomyocardial biopsy [8].

The new approach in the evaluation of most cardiac pathologies is multimodality imaging, or otherwise said, the capability of combining different imaging techniques in order to obtain, in the most efficient manner, an accurate diagnosis and capable of offering therapy guidance or predicting outcomes $[9,10]$.

\section{Echocardiography}

In most cardiovascular diseases, echocardiography occupies an extremely important role, being an indispensable imaging method in cardiomyopathies assessment. The main advantages are that it is a non-irradiating and noninvasive imaging method, has the ability to provide realtime images, a very good resolution and is also accessible, portable and cost effective [11].

Echocardiography is able to provide information about the cardiac function and structure (including the valves); concerning the size of cardiac chambers, wall thickness and motion abnormalities, LVEF, diastolic function, $\mathrm{SV}$ and $\mathrm{CO}$ and also pulmonary artery or intracardiac pressure estimations can be obtained [11].

Nowadays, the echocardiographic techniques that are used in cardiomyopathies assessment, are: two and three-dimensional echocardiography (2DE and 3DE), color and spectral Doppler, 2D strain, torsion, contrast and tissue Doppler imaging (TDI).

$2 \mathrm{DE}$ is part of routine investigation of patients with CM, especially in left ventricle assessment, helping to determine the size of cardiac chambers, the morphology and function, including LVEF. In standard practice echocardiography, a 17-segment model is used for a more accurate localization of the affected region $[11,12]$.

The most commonly used method in LVEF quantification is the Simpson biplane method, using standard views (2 and 4-chamber) [12,13]. The measurements obtained can predict adverse cardiovascular events such as arrhythmias, heart failure (HF) or death [14]. LVEF can be obtained also using another imaging method - CMR. The values obtained using these two techniques correlate well, but echocardiography tends to underestimate the left ventricular volumes owing to limitations of image quality and of geometric assumptions [15]. 


\section{Cardiac magnetic resonance imaging General information}

Cardiovascular magnetic resonance imaging or cardiac MRI (CMR) is a non-invasive and nonirradiating imaging method, complementary to cardiac ultrasound. This imaging method is considered the gold standard in assessing the structure and function of cardiac chambers, having an important role in the evaluation of cardiomyopathies. Conventional MRI sequences are adapted for cardiac imaging by using ECG gating and high temporal resolution protocols [16].

The possibility of using gadolinium contrast agents with CMR and the analysis of the contrast enhancement in the late stages after injection (LGE), have further increased the ability to examine the myocardial tissue, in particular myocardial fibrosis, which, according to the most recent studies, represents the necessary substrate for the majority of malignant ventricular arrhythmias (VA) [17].

Although it is not the imaging method of first intention in the assessment of cardiovascular pathology, it is superior to echocardiography in the assessment of cardiac volumes, LVEF, in the analysis of cardiac wall dyskinesia and it contributes to the characterization of myocardial tissue $[17,18]$. Echocardiography can be limited by poor acoustic windows, making it difficult to obtain a complete examination of the heart. Not having this shortcoming, CMR may help diagnose diseases otherwise undetectable by echocardiography. Therefore, it is considered the method of choice even in the assessment of congenital heart diseases [19].

The current techniques of CMR focus on the assessment of the function and structure of the left ventricle, characterization of myocardial tissue (in particular myocardial fibrosis) and detection of thrombus [19].

A comprehensive and useful CMR examination for cardiomyopathies should include T1- and T2-weighted sequences, cine- sequences (function assessment), myocardial enhancement in perfusion imaging and detection of fibrotic or infarcted areas analyzing the LGE [20].

In everyday practice, every CMR investigation includes a quantitative assessment of cardiac chambers size and ventricular function [21]. The functional assessment consists in realizing cine- sequences in short and long axis, 2, 3 and 4 chamber series, to achieve full coverage of the left ventricle with a slice thickness that varies between 6 to $8 \mathrm{~mm}$, thus obtaining a 3-dimensional (3D) volumetric structure for analysis [7]. One can obtain very accurate and reproducible information regarding the ejection fraction, ventricular mass and volumes [22]. The most used cinesequence is steady state free precession (SSFP) which offers a great contrast-to-noise ratio between the bright blood pool and the dark myocardium [23].

When using CMR it is no longer necessary to make geometric assumptions, like in the case of $2 \mathrm{D}$ echocardiography, especially when the ventricles are deformed by MI or CMP, since the measurements are obtained from a 3D data set [7].

\section{CMR techniques}

\section{Late Gadolinium enhancement (LGE)}

It is obtained after contrast administration (0.1-0.2 $\mathrm{mmol} / \mathrm{kg}$ of $\mathrm{Gd}$ ), making a late acquisition after a delay of 10-15 minutes [7]. This CMR technique uses ECG gating for adequate relaxation between inversion pulses, with data being obtained at each heartbeat [24]. The used sequences are called inversion recovery pulse sequence (Figure 1) by which the difference between the normal and pathological myocardium signal may be increased by $500-1000 \%$ [25].

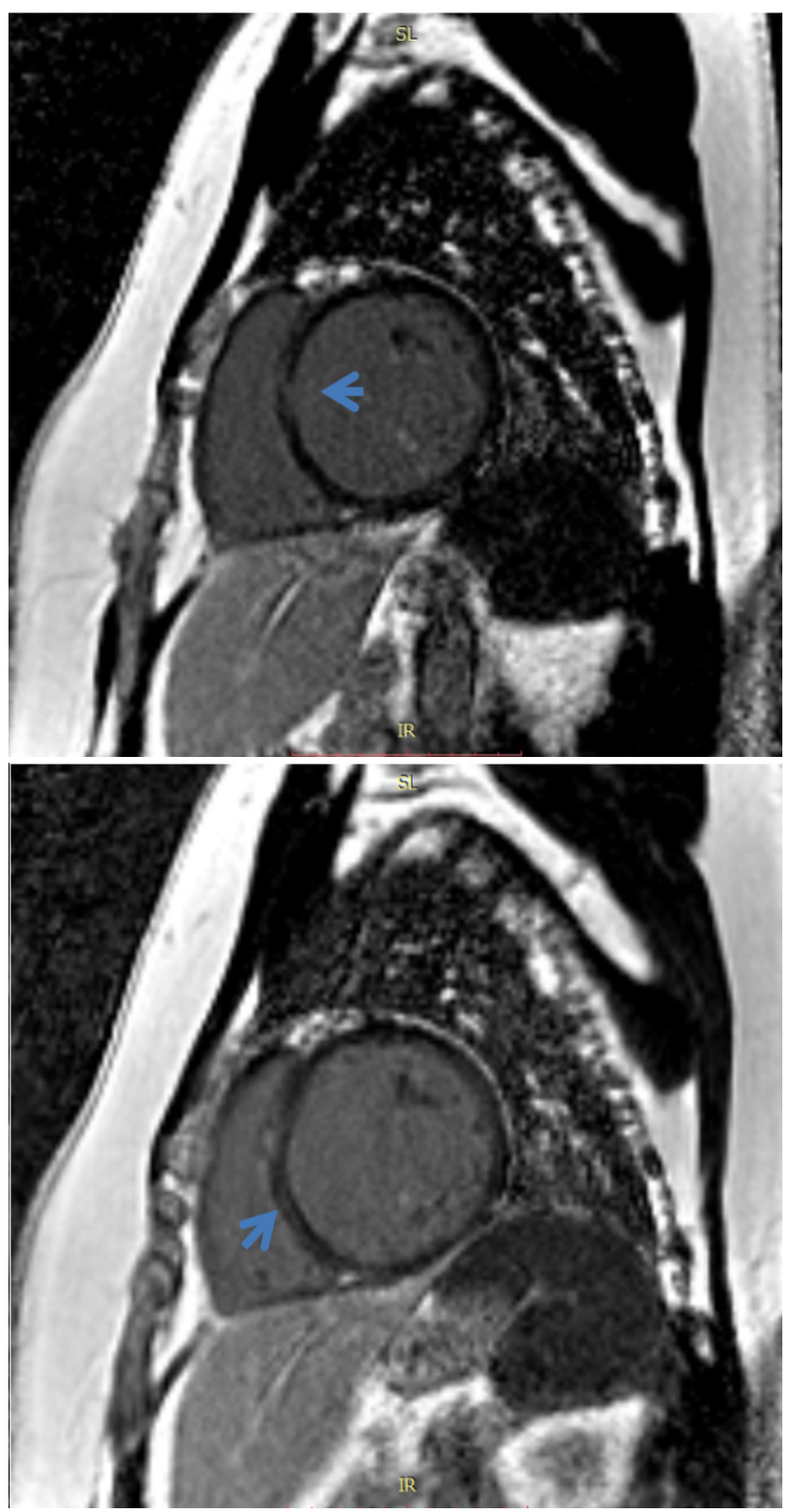

Figure 1. Cardiac MRI. Delayed PSIR sequence, short axis view. Midwall septal fibrosis (late gadolinium enhancement) in a patient with dilated cardiomyopathy. 
LGE will appear as hypersignal regions, referring to areas of inflammation, necrosis or fibrotic tissue (scar) that have a prolonged retention of Gd compared to normal tissue, being particularly useful in the assessment of myocardial lesions. Another interesting fact is that there is no contrast retention in areas with myocardial reversible injuries compared to areas of myocardial necrosis or fibrotic tissue. Also, there is a significant overlap between LGE and infarction as defined by histology, all this demonstrated by Kim et al [26,27].

This CMR technique is very useful in making the differential diagnosis between ischemic (ICM) and nonischemic (NICM) etiology of cardiomyopathies, the site being essential: subendocardic in ICM, with vascular distribution and varied pattern, without vascular distribution in NICM. Also, in patients affected by cardiomyopathies, LGE is a good predictor of adverse cardiovascular events [7]. For example, in patients with NICM, the presence of LGE was associated with odds ratios of 3.3 for mortality and 5.3 for SCD [28]. Or, more specifically, in patients affected by DCM, who have an LVEF $<35 \%$, the presence of LGE was associated with an 8-fold increase in heart failure and cardiac death [29].

\section{T1 and T2 mapping}

Due to technological progress in recent years, CMR imaging techniques allow the comparison between quantified myocardial parameters and normal reference values, the acquisitions being made in the same conditions (scan time, scanner type, contrast agent) [30].

Many diffuse disease of the myocardium, including cardiomyopathies, have associated cardiac MRI studies considered as normal, because there are no changes in motility, signal intensity or LGE. However, changes of the $\mathrm{T} 1$ and $\mathrm{T} 2$ relaxation times may occur. There are some specialized pulse sequences in CMR, able to estimate $\mathrm{T} 1$ and $\mathrm{T} 2$ values and to create, for each of them, color anatomic overlay maps, after acquiring a series of images of the same region of the myocardium, in which the pixel values represent the T1/T2 in each voxel (rather than a signal intensity in arbitrary units) [31].

An important CMR parameter is $\mathrm{T} 1$ relaxation time, also called longitudinal relaxation time, that can be measured before (native T1 - water signal from extracellular space and myocytes) and after contrast infusion (useful in calculating the extracellular volume fraction - ECV) [32]. In the case of patients that have contraindication for contrast administration, such as stage 4 or 5 of chronic kidney disease, native T1 is also very useful [24]. The native $\mathrm{T} 1$ relaxation time value is constant and specific for each tissue, being influenced by changes in water composition or of local molecular environment. Some of the myocardial diseases that can change $\mathrm{T} 1$ are infiltrative diseases (hemosiderosis, amyloidosis or Fabry disease), edema, inflammation and diffuse myocardial fibrosis [7]. These CMR imaging techniques can help in diagnosing some cardiomyopathies that are known to have high native T1 and high ECV.

By the T1 mapping technique parametric, colored anatomic maps that are generated from a series of images acquired with different $\mathrm{T} 1$ weighting are obtained so that each pixel can be assigned a T1 value. The T1 maps obtained permit a quantitative and visual interpretation. The most assessed T1 mapping sequence is considered MOLLI sequence (modified Look Locker inversion recovery), which overcomes the limitations of motion and prolonged acquisition time [33].

Another parameter of CMR imaging called T2 relaxation time referred to as transverse relaxation time, can also be used to distinguish the abnormal myocardial tissue from the normal one. The values are acquired using SSFP sequences [7]. The main cause for longer T2weighted imaging is the increase in the water content of myocardial tissues; therefore, the main entity that causes an elevated T2 is myocardial edema. Thus, an increased T2 relaxation time can be seen in patients with acute myocarditis, acute myocardial infarction, sarcoidosis, cardiac allograft rejection or stress cardiomyopathy [24].

In the T2 mapping technique a parametric image is obtained based on the $\mathrm{T} 2$ value calculated for each voxel. Then, the T2 maps, visualized on a grey or color scale, can be quantitatively analyzed by the investigator which can make measurements in the regions of interest [33].

Nowadays, T1 mapping is considered similar to a non invasive biopsy of the heart, but further studies are needed before a standardization can be adopted. Among the main strengths of this method are the facts that there is not always a need for contrast administration and the high reproducibility and clinical applicability. In the future, T1 mapping is expected to have a prognostic value and be able to help monitoring different types of myocardial diseases [7].

\section{CMR as a tool for establishing the etiology of cardiomyopathies}

Assessment of late gadolinium enhancement is a CMR technique very useful in making the differential diagnosis between cardiomyopathies, especially between ischemic and non-ischemic etiology. In ICM, the late contrast retention starts always in the subendocardial region, with or without transmural extension and follows a vascular distribution compared to nonischemic cardiomyopathies (NICM) where there is no coronary distribution, existing a varied pattern such as subepicardial, midwall, patchy, localized or diffuse [7].

In NICM, this technique permits the assessment of the myocardial tissue and thus the diagnosis of a variety of pathologies such as non-compaction cardiomyopathy, myocarditis, sarcoidosis, amyloidosis, haemochromatosis, Chagas disease and Fabry disease [34]. 


\section{Ischemic cardiomyopathy - CMR in acute myocardial infarction (AMI)}

The signs of an AMI, as illustrated by the CMR examination, are: high signal intensity on T2 and STIR (Figure 2), subendocardial or transmural extent suggesting edema (low specificity); LGE in vascular territories (cardiac segments); perfusion disorders in early postcontrast phases; assessment of parietal dyskinesia. CMR provides the positive diagnosis, including in the early phase (first hour after the onset), assessing the extent and age of the ischemic event.

CMR can also detect AMI sequelae: myocardial wall thinning $(<7 \mathrm{~mm})$, late gadolinium enhancement (,white is dead"), complications like aneurysmal dilatation, intracavitary thrombosis (allowing the measurement of function even in this cases) or myocardial calcifications. Also, this advanced imaging modality, is capable of making the differential diagnosis with myocarditis or Takotsubo cardiomyopathy. It is also useful in the therapeutic follow-up, influencing the therapeutic decision by assessing the viability of the affected myocardium [35].

According to numerous studies, important markers of the non viability of the myocardium, therefore with a low chance of contractile recovery after revascularization, are LV wall thickness less than $5.5 \mathrm{~mm}$ at end-diastole [36] and transmural infarcts (transmural extent of LGE $>50 \%$ were nonviable) [37]. Also, the contractile reserve can be assessed by cine imaging of the left ventricle after administration of low dose dobutamine, being able to predict areas of functional recovery with revascularization, offering the highest specificity $(91 \%)$ and positive predictive value (93\%) [38,39].
A well known complication of coronary reperfusion therapy after an AMI is microvascular obstruction (MVO) and intramyocardial hemorrhage.

MVO represents areas of irreversible myocardial infarction. Its presence on CMR imaging has been associated with a poor prognosis, correlated with adverse LV remodelling and dysfunction. Also, these patients are more likely to experience future major adverse cardiovascular events [40].

MVO evaluation by CMR imaging implies its detection, quantification and evolution [41]. A recommended CMR protocol starts with perfusion sequences, where MVO appears as a focal low signal lesion within an area of early enhancing infarcted myocardium. There are similar appearances on both early and late gadolinium-enhancement sequences (EGE and LGE), the true area of MVO being seen in delayed phases (Figure 3), of smaller size than first-pass perfusion (slow diffusion of Gd over time around the periphery of the noreflow site) [40].

Intramyocardial hemorrhage is caused by the disruption to the microvasculature after reperfusion therapy. CMR assessment implies T2-weighted and T2* sequences [41].

The appearance of hemorrhage on MRI is based upon the paramagnetic effects of hemoglobin degradation products [42]. In the acute phase, on T2-weighted sequence, appears as low signal regions surrounded by elevated signal intensity representing myocardial edema. A more useful MRI sequence is T2*, with high sensitivity in detecting magnetic field non-homogeneity caused by iron deposits and blood products for detecting hemorrhage as low signal areas [41].
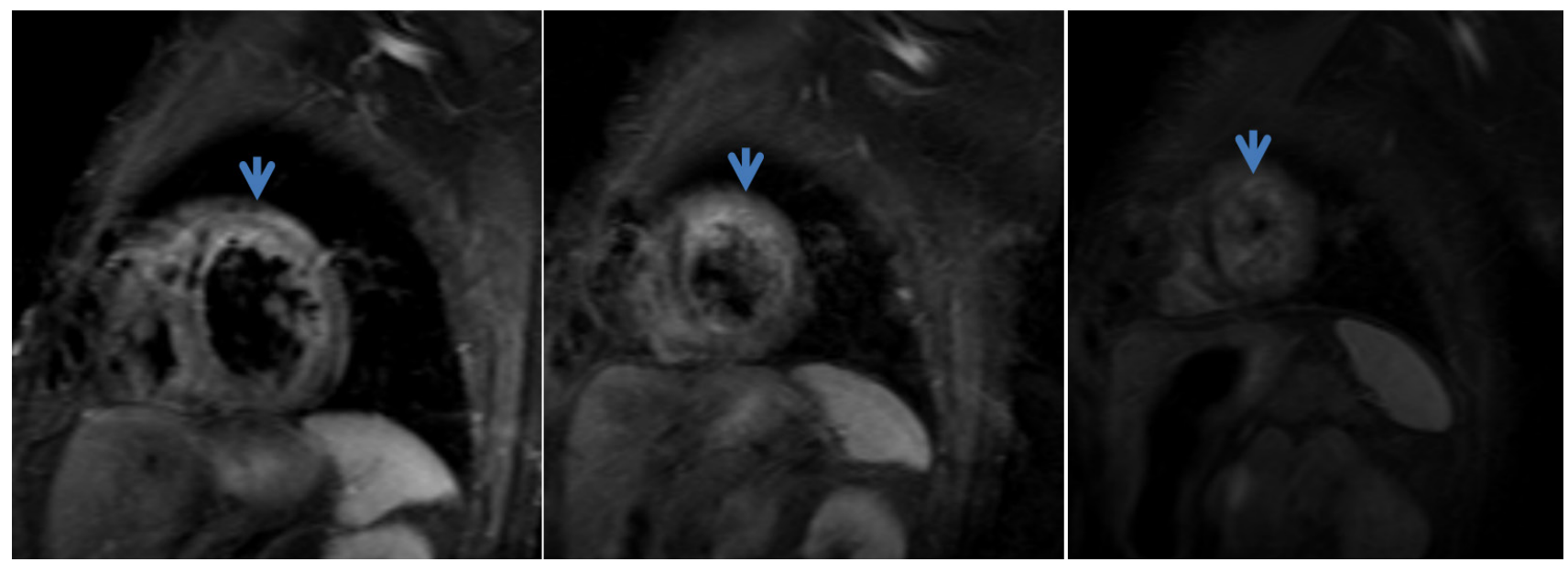

Figure 2. Cardiac MRI. Turbo inversion recovery magnitude (TIRM) sequence, short axis view. Edema in the anterior wall and apical region. 

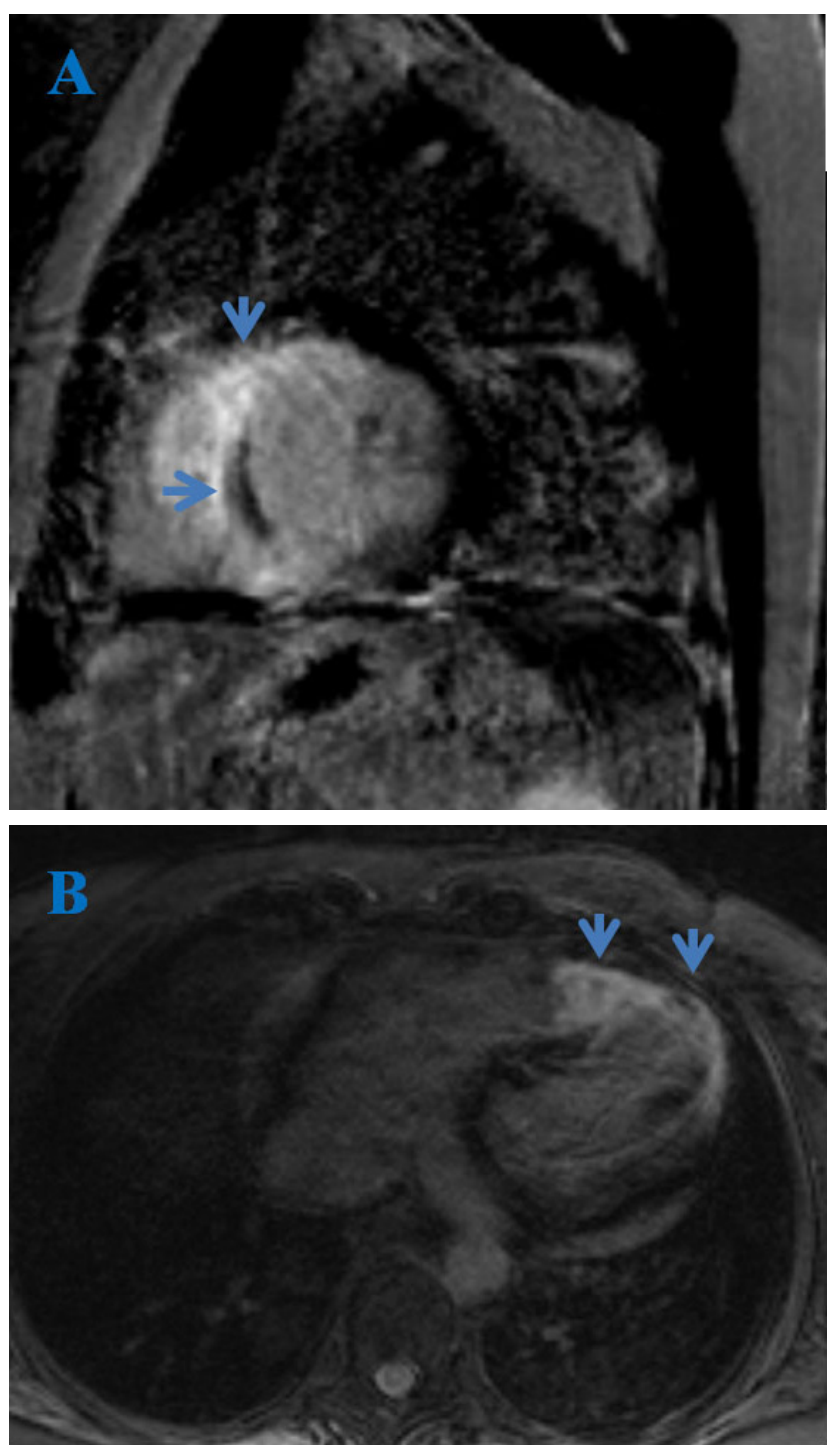

Figure 3. Cardiac MRI. Delayed PSIR sequence, short axis view (A) and $4 \mathrm{CH}$ view (B). Acute myocardial infarction in LAD distribution with non enhancing foci, highly suspicious for microvascular obstruction and intra-myocardial hemorrhage.

\section{Nonischemic cardiomyopathies}

In nonischemic cardiomyopathies CMR has proven its usefulness by being capable of tissue characterization and thus, differentiating the etiologies (late gadolinium enhancement or T1 mapping for certain hypertrophic and infiltrative cardiomyopathies) [42].

\section{Dilated cardiomyopathy (DCM)}

Dilated cardiomyopathy (DCM) is defined as left ventricular chamber dilation, thin-walled, with decreased systolic function (Figure 4); it can be the end-stage manifestation of numerous disorders of non-ischemic etiology such as alcohol or cocaine chronic use, endocrine disorders such as growth hormone excess, thyroid diseases, diabetes mellitus, pheochromocytoma or myocarditis [24].

An important component of DCM is myocardial fibrosis, which CMR is capable of detecting. It is present in two forms: irreversible replacement fibrosis (as LGE) and diffuse interstitial fibrosis (detected on $\mathrm{T}_{1}$ mapping) [43]. If LGE is present, the most common pattern is in the midwall of the interventricular septum; however, a minority of patients could have subendocardial LGE and may be classified as ischemic (misclassification) [44]. Midwall fibrosis proved to be an independent predictor of mortality and morbidity, having a similar outcome to ischemic disease [45]; also, according to the most recent studies [46], represents the underlying cause for the majority of malignant ventricular arrhythmias (AV) [47].

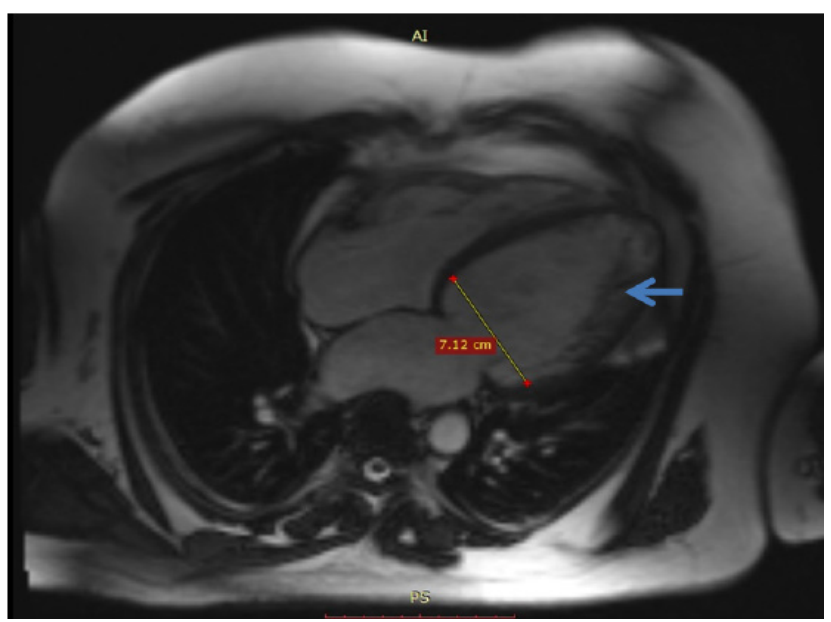

Figure 4. Cardiac MRI. True FISP image, 4-chamber view. Dilated cardiomyopathy and non-compaction of the left ventricle.

\section{Hypertrophic cardiomyopathy (HCM)}

This is the most common genetic heart disease, with a prevalence of 1 in 200 to 300 in the general population, caused by a mutation in genes encoding any of the cardiac contractile proteins [48]. Echocardiography is the imaging technique used for screening purposes. CMR is more sensitive in identifying unusual or apical sites of hypertrophy. Also, it is the gold standard in assessing LV mass, this being an important prognostic factor (higher mass - worse outcome) [49].

The majority of patients with HCM have a LGE patchy pattern. This pattern can be visualized especially when there is a significant hypertrophy, at the right ventricular septal insertion sites.

The presence of late gadolinium enhancement in HCM patients (Figure 5) has been associated with increased risk of ventricular and re-entrant tachycardias and SCD [50].

A meta-analysis of four studies performed on 1063 patients, followed for an average of 3.1 years, demonstrated that the presence of LGE had an odds ratio of 5.7 for heart failure death, 2.9 for cardiac death and 4.5 for all-cause mortality [51]. 


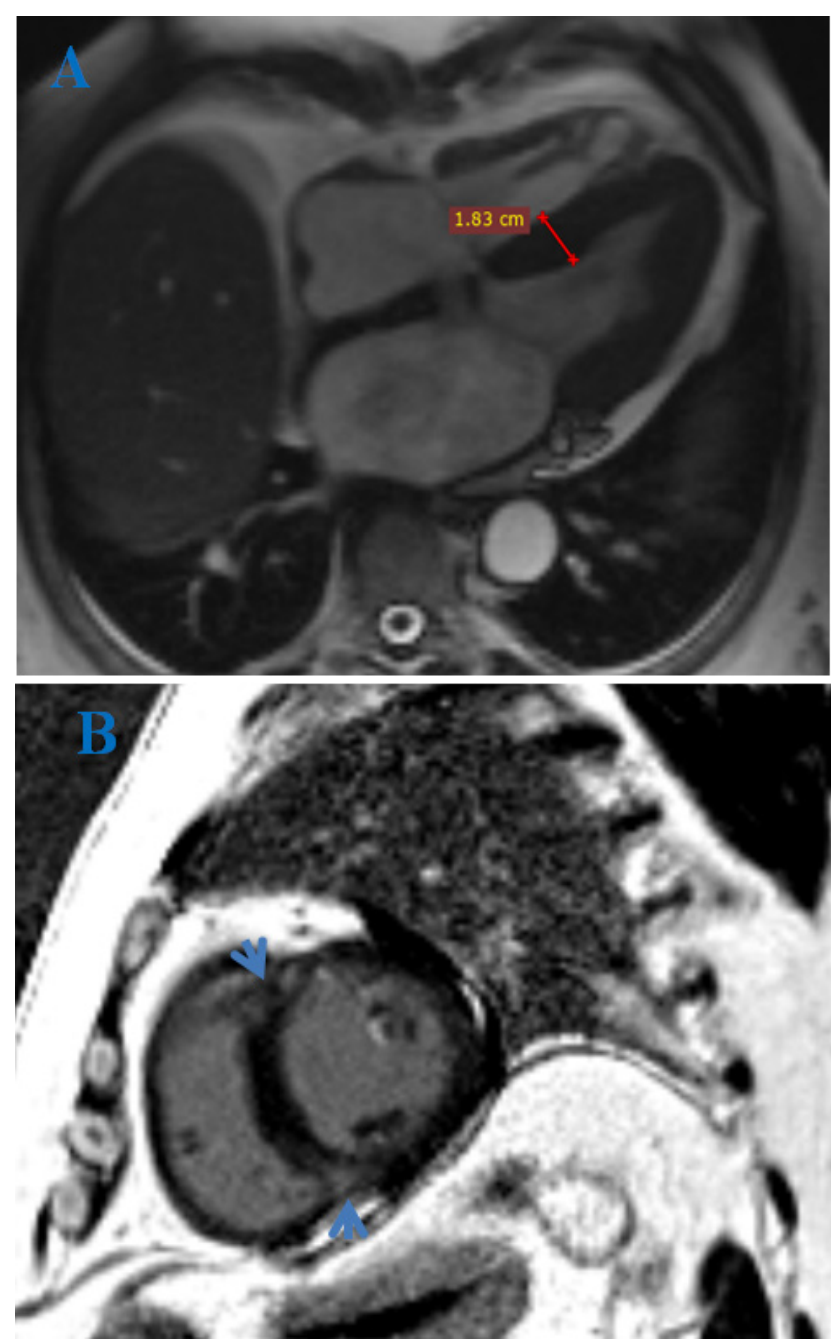

Figure 5. Cardiac MRI. True FISP image, 4-chamber view (A) and delayed PSIR sequence, short axis view (B). Subepicardial fibrosis (arrows) in a patient with hypertrophic cardiomyopathy.

\section{Myocarditis}

In both the pediatric and adult population, it has a primarily viral origin. A characteristic finding revealed by CMR is late gadolinium enhancement in the midwall and subepicardial of the left ventricle [52].

Other MRI signs, suggested by different studies, are early enhancement of the myocardium on T1-weighted sequence (as inflammation marker) or hypersignal on the sagittal T2-STIR sequence (myocardial edema) (Figure 6) [53]. In 2009, the Lake Louise consortium suggested an ideal diagnostic strategy based on the positivity ( 2 of 3 ) of these 3 entities: early enhancement ratio, late gadolinium enhancement and increased T2 signal [54].

Nowadays, the early enhancement ratio has a less important role and the $\mathrm{T} 2-\mathrm{W}$ sequence has been replaced by $\mathrm{T} 2$ mapping. Another useful tool is T1 mapping (native and post-contrast). According to the most recent studies, a combination between LGE, T1 and T2 mapping may be the best way to identify acute myocarditis [47]. A study of 104 patients and 21 controls, that used LGE and T1-mapping, demonstrated an overall accuracy of $90 \%$ [55].

In chronic myocarditis, another study of 67 patients, showed that late gadolinium enhancement alone has a better accuracy than T1-mapping ( $94 \%$ vs. $84 \%$ ), with an increased accuracy when these two techniques are combined $(98 \%$ overall accuracy) [56].

In the pediatric population, accurate diagnosis of myocarditis is challenging due to the variability of symptoms. Especially newborns, infants and immunocompromised patients are susceptible for developing myocarditis. The most common infections involved are those with adenovirus and enterovirus. It is an important cause of morbidity and mortality that may lead to acute heart failure, CMD and SCD [57].
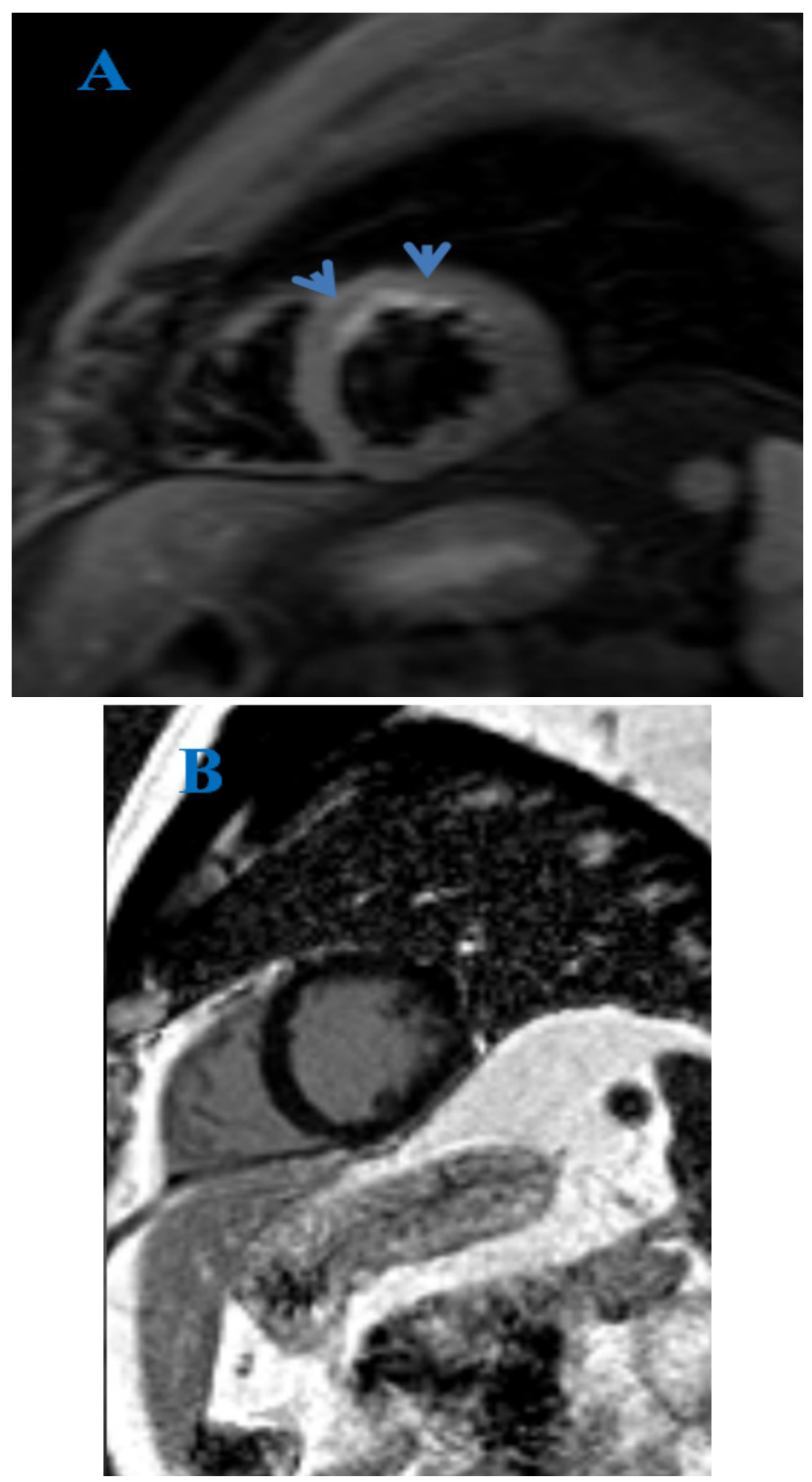

Figure 6. Cardiac MRI. Turbo inversion recovery magnitude (TIRM) sequence, short axis view (A) and delayed PSIR sequence, short axis view (B). Edema in anterior and septal walls (arrows). Normal myocardial perfusion. No LGE. Findings suggestive for acute myocarditis. 
Regarding MRI findings, there are few studies regarding its utility in pediatric acute myocarditis. There are the same CMR signs as in adults, on T2 and post-contrast sequences, such as: signal increase on T2-weighted images at early disease or normal signal in the healing phase; early and late gadolinium enhancement. Other associated signs could be left systolic dysfunction (common), increased LV dimensions, segmental wall motion abnormalities and pericardial effusion. A multimodal approach is needed for an accurate diagnosis [58].

\section{Amyloidosis}

Amyloidosis is a disease that is characterized by extracellular deposition of insoluble fibrillar proteins, which can affect a single organ or can be systemic. The cardiac involvement is variable, depending on the type of amyloidosis: primary (up to 50\%), familial (between 10 and $50 \%)$ and secondary $(<5 \%)[50]$.

The signs of amyloid infiltration evidenced by transthoracic echocardiogram are decreased cavity size, increased left ventricle wall thickness, pericardial effusion, biatrial enlargement, diastolic dysfunction or by ECG like low voltage in the limb leads. However, CMR has a greater sensitivity and specificity compared to TTE, showing a global LGE in the subendocardial region [53].

CMR can be used also to differentiate between primary and familial amyloidosis [24]. A study conducted on 97 patients, 46 patients of them with primary amyloidosis and 51 with familial amyloidosis, analyzed the presence of LGE, revealing that late gadolinium enhancement is more extended in the familial form (present in $90 \%$ of cases vs $37 \%$ in the primary form) [59].

\section{Sarcoidosis}

The exact prevalence of cardiac involvement is still not established, varying depending on the study between $5 \%$ to $20 \%$ of patients affected by this disease [60].

Current guidelines for diagnosis of cardiac involvement in sarcoidosis are based on histological evidence, LGE on CMR (high sensitivity and specificity), ECG and echocardiographic abnormalities [50].

On CMR, the specific pattern of this disease consists of LGE in midwall or subepicardial region, especially in the basal and lateral segments of the LV. In a minority of cases, LGE can be present subendocardial or transmural. In addition, CMR can detect also edema or motion abnormalities. The presence of late gadolinium enhancement is associated with a higher risk of major adverse cardiac events and SCD [61].

\section{Arrhythmogenic right ventricular cardiomyopathy}

ARVC is an inherited disease characterized by fibrofatty replacement of the right ventricular free wall (in a minority of cases can affect the left ventricle) [50].

CMR is considered the gold standard examination in the evaluation of ARCV. There are some difficulties to perform this in children or in patients with arrhythmias (cardiac gating errors) [62]. The RV function can be appreciated. The main findings include RV dilation, aneurysm formation, hypokinesia of the RV, fatty or fibrofatty infiltration (high signal on T1 with suppression on FAT-SAT sequences).

\section{Non-compaction cardiomyopathy}

Non-compaction cardiomyopathy or left ventricular hypertrabeculation (Figure 7), is a congenital disease characterized by a ratio of 2.3 at end-diastole between noncompacted and compacted myocardium [63]. Additional CMR findings: left ventricle thrombus or systolic dysfunction [24].

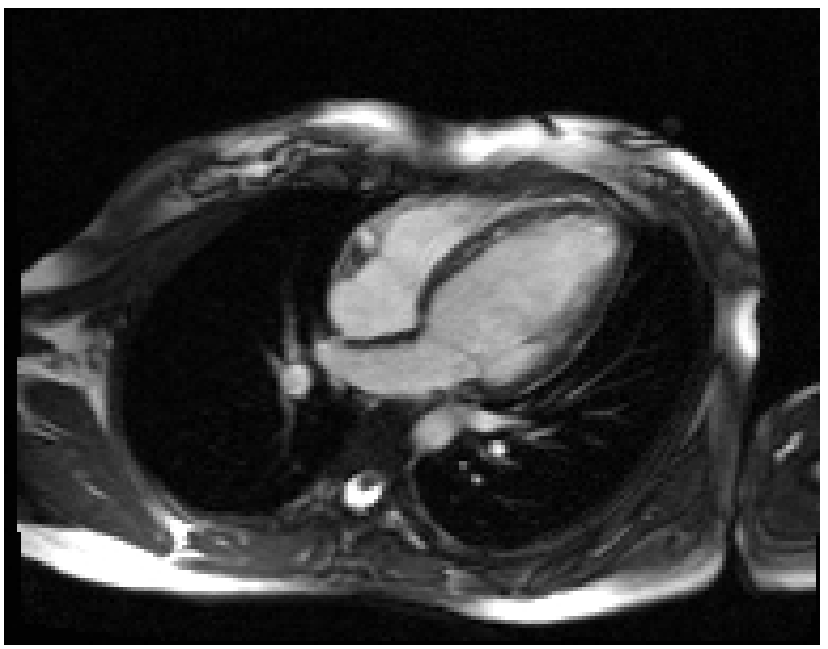

Figure 7. Cardiac MRI. True FISP image, 4-chamber view. Noncompaction of the left ventricle (arrows).

\section{Takotsubo cardiomyopathy}

This is a stress-related CM, also known as "broken heart syndrome" or apical ballooning syndrome. It consists of a transient left ventricular ballooning and occurs after emotional stress, without any signs of coronary artery disease (CAD) [50].

CMR is able to depict myocardial edema without LGE (important feature), apical ballooning and wall motion dyskinesia [64], thus making the differential diagnosis with acute myocardial infarction (subendocardial LGE) and myocarditis (patchy LGE) [50].

\section{Limitations and contraindications of CMR}

Among the limitations are included factors that can influence image quality such as: partial volume effect, presence of ventricular arrhythmias during acquisition, lack of adequate apnea, variability of kinetics of the contrast agent which can result in an inaccurate scarring characterization. There are difficulties in examining claustrophobic patients, certain obese patients, patients with acute pathologies that can not cooperate. Also, the method implies higher costs and lower availability compared with echocardiography [65].

The contraindications of a CMR examination include: patients with pacemaker, old cardiac prostheses, 
with uncertainty about safety in patients with metallic fragments present within soft tissues. It is also contraindicated in patients with articular metal prostheses or osteosynthesis materials, aneurysm clips or certain vascular stents. Another important contraindication is the administration of Gd-based contrast agents in patients with severe renal impairment, with a glomerular filtration rate (GFR) below $30 \mathrm{~mL} / \mathrm{min} / 1.73 \mathrm{~m} 2$, because there is a risk of nephrogenic systemic fibrosis [34].

\section{Conclusions}

Cardiac magnetic resonance imaging is a crucial tool, with an efficiency that has been demonstrated, capable of providing the differential diagnosis between ischemic and nonischemic cardiomyopathy and of establishing the etiology in the nonischemic type. In addition, CMR findings have a prognostic value, may guide patient management and, if necessary, can evaluate treatment response. Therefore, CMR should be a part of the routine investigation in various cardiomyopathies.

\section{References}

1. Elliott P, Andersson B, Arbustini E, Bilinska Z, Cecchi F, Charron $\mathrm{P}$, et al. Classification of the cardiomyopathies: a position statement from the European Society Of Cardiology Working Group on Myocardial and Pericardial Diseases. Eur Heart J. 2008;29:270-276.

2. McKenna WJ, Maron BJ, Thiene G. Classification, Epidemiology, and Global Burden of Cardiomyopathies. Circ Res. 2017;121:722-730.

3. Maron BJ, Towbin JA, Thiene G, Antzelevitch C, Corrado D, Arnett D, et al. Contemporary definitions and classification of the cardiomyopathies: an American Heart Association Scientific Statement from the Council on Clinical Cardiology, Heart Failure and Transplantation Committee; Quality of Care and Outcomes Research and Functional Genomics and Translational Biology Interdisciplinary Working Groups; and Council on Epidemiology and Prevention. Circulation. 2006;113:1807-1816.

4. Dec GW, Fuster V. Idiopathic dilated cardiomyopathy. N Engl J Med. 1994;331:1564-1575.

5. Towbin JA, Lowe AM, Colan SD, Sleeper LA, Orav EJ, Clunie S, et al. Incidence, causes, and outcomes of dilated cardiomyopathy in children. JAMA. 2006;296:1867-1876.

6. Rosamond W, Flegal K, Friday G, Furie K, Go A, Greenlund $\mathrm{K}$ et al. Heart disease and stroke statistics - 2007 update: a report from the American Heart Association Statistics Committee and Stroke Statistics Subcommittee [published correction appears in Circulation. 2007;115:e172]. Circulation. 2007;115:e69-e171.

7. Jan MF, Tajik AJ. Modern Imaging Techniques in Cardiomyopathies. Circ Res. 2017;121:874-891.

8. Wei S, Fu J, Chen L, Yu S. Performance of cardiac magnetic resonance imaging for diagnosis of myocarditis compared with endomyocardial biopsy: a meta-analysis. Med Sci Monit. 2017;23:3687-3696.

9. Ngiam N, Chew N, Chai P, Poh KK. Multimodality Cardiac
Imaging in the Evaluation of a Patient with Near-Fatal Arrhythmia. Ann Acad Med Singapore. 2019;48:39-41.

10. Arbustini E, Narula N, Dec GW, Reddy KS, Greenberg B, Kushwaha S, et al. The MOGE(S) classification for a phenotype-genotype nomenclature of cardiomyopathy: endorsed by the World Heart Federation. J Am Coll Cardiol. 2013;62:2046-2072.

11. Lang RM, Badano LP, Mor-Avi V, Afilalo J, Armstrong A, Ernande L, et al. Recommendations for cardiac chamber quantification by echocardiography in adults: an update from the American Society of Echocardiography and the European Association of Cardiovascular Imaging. J Am Soc Echocardiogr. 2015;28:1-39.e14.

12. Lang RM, Bierig M, Devereux RB, Flachskampf FA, Foster E, Pellikka PA, et al. Recommendations for chamber quantification: a report from the American Society of Echocardiography's Guidelines and Standards Committee and the Chamber Quantification Writing Group, developed in conjunction with the European Association of Echocardiography, a branch of the European Society of Cardiology. J Am Soc Echocardiogr. 2005;18:1440-1463.

13. Otterstad JE. Measuring left ventricular volume and ejection fraction with the biplane Simpson's method. Heart. 2002;88:559-560.

14. St John Sutton M, Pfeffer MA, Moye L, Plappert T, Rouleau JL, Lamas G, et al. Cardiovascular death and left ventricular remodeling two years after myocardial infarction: baseline predictors and impact of long-term use of captopril: information from the Survival and Ventricular Enlargement (SAVE) trial. Circulation. 1997;96:3294-3299.

15. Demir H, Tan YZ, Kozdag G, Isgoren S, Anik Y, Ural D, et al. Comparison of gated SPECT, echocardiography and cardiac magnetic resonance imaging for the assessment of left ventricular ejection fraction and volumes. Ann Saudi Med. 2007;27:415-420.

16. Rijnierse MT, van der Lingen AL, Weiland MT, de Haan S, Nijveldt R, Beek AM, et al. Clinical Impact of Cardiac Magnetic Resonance Imaging Versus EchocardiographyGuided Patient Selection for Primary Prevention Implantable Cardioverter Defibrillator Therapy. Am J Cardiol. 2015; 116:406-412.

17. Nazarian S, Bluemke DA, Lardo AC, Zviman MM, Watkins SP, Dickfeld TL, et al. Magnetic resonance assessment of the substrate for inducible ventricular tachycardia in nonischemic cardiomyopathy. Circulation. 2005;112:2821-2825.

18. Hsia HH, Marchlinski FE. Characterization of the electroanatomic substrate for monomorphic ventricular tachycardia in patients with nonischemic cardiomyopathy. Pacing Clin Electrophysiol. 2002;25:1114-1127.

19. Gonzalez JA, Kramer CM. Role of imaging techniques for diagnosis, prognosis and management of heart failure patients: cardiac magnetic resonance. Curr Heart Fail Rep. 2015;12:276-283.

20. West AM, Kramer CM. Cardiovascular magnetic resonance imaging of myocardial infarction, viability, and cardiomyopathies. Curr Probl Cardiol. 2010;35:176-220.

21. Hundley WG, Bluemke D, Bogaert JG, Friedrich MG, Higgins CB, Lawson MA, et al. Society for Cardiovascular Magnetic Resonance guidelines for reporting cardiovascular magnetic resonance examinations. J Cardiovasc Magn Reson. 2009;11:5. 
22. Isbell DC, Kramer CM. Cardiovascular magnetic resonance: structure, function, perfusion, and viability. J Nucl Cardiol. 2005;12:324-336.

23. Miller S, Simonetti OP, Carr J, Kramer U, Finn JP. MR imaging of the heart with cine true fast imaging with steadystate precession: influence of spatial and temporal resolutions on left ventricular functional parameters. Radiology. 2002;223:263-269.

24. Kramer CM. Role of Cardiac MR Imaging in Cardiomyopathies. J Nucl Med. 2015;56 Suppl 4:39S-45S.

25. Simonetti OP, Kim RJ, Fieno DS, Hillenbrand HB, Wu E, Bundy JM, et al. An improved MR imaging technique for the visualization of myocardial infarction. Radiology. 2001;218:215-223.

26. Kim RJ, Fieno DS, Parrish TB, Harris K, Chen EL, Simonetti $\mathrm{O}$, et al. Relationship of MRI delayed contrast enhancement to irreversible injury, infarct age, and contractile function. Circulation. 1999;100:1992-2002.

27. Rehwald WG, Fieno DS, Chen EL, Kim RJ, Judd RM. Myocardial magnetic resonance imaging contrast agent concentrations after reversible and irreversible ischemic injury. Circulation. 2002;105:224-229.

28. Kuruvilla S, Adenaw N, Katwal AB, Lipinski MJ, Kramer CM, Salerno M. Late gadolinium enhancement on cardiac magnetic resonance predicts adverse cardiovascular outcomes in nonischemic cardiomyopathy: a systematic review and meta-analysis. Circ Cardiovasc Imaging. 2014;7:250-258.

29. Wu KC, Weiss RG, Thiemann DR, Kitagawa K, Schmidt A, Dalal D, et al. Late gadolinium enhancement by cardiovascular magnetic resonance heralds an adverse prognosis in nonischemic cardiomyopathy. J Am Coll Cardiol. 2008;51:2414-2421.

30. Kim PK, Hong YJ, Im DJ, Suh YJ, Park CH, Kim JY, et al. Myocardial T1 and T2 Mapping: Techniques and Clinical Applications. Korean J Radiol. 2017;18:113-131.

31. Burt JR, Zimmerman SL, Kamel IR, Halushka M, Bluemke DA. Myocardial T1 mapping: techniques and potential applications. Radiographics 2014;34:377-395.

32. Moon JC, Messroghli DR, Kellman P, Piechnik SK, Robson MD, Ugander M, et al. Myocardial T1 mapping and extracellular volume quantification: a Society for Cardiovascular Magnetic Resonance (SCMR) and CMR Working Group of the European Society of Cardiology consensus statement. J Cardiovasc Magn Reson. 2013;15:92.

33. Hamlin SA, Henry TS, Little BP, Lerakis S, Stillman AE. Mapping the future of cardiac MR imaging: case-based review of T1 and T2 mapping techniques. Radiographics. 2014;34:1594-1611.

34. Ponikowski P, Voors AA, Anker SD, Bueno H, Cleland JGF, Coats AJS, et al. The Task Force for the diagnosis and treatment of acute and chronic heart failure of the European Society of Cardiology (ESC). European Heart Journal. 2016;37:2129-2200.

35. Manole S. Sindromul coronarian acut [Acute coronary syndrome]. In: SRIM - Radiologie si Imagistica Medicala - Indrumator de studiu pentru pregatirea in specialitate [Medical radiology and imaging - Guide for specialist training] Vol. I. Bucuresti: Ed. Medicala 2015:544-546.

36. Shah DJ, Kim HW, James O, Parker M, Wu E, Bonow $\mathrm{RO}$, et al. Prevalence of regional myocardial thinning and relationship with myocardial scarring in patients with coronary artery disease. JAMA. 2013;309:909-918.

37. Kim RJ, Wu E, Rafael A, Chen EL, Parker MA, Simonetti $\mathrm{O}$, et al. The use of contrast-enhanced magnetic resonance imaging to identify reversible myocardial dysfunction. $\mathrm{N}$ Engl J Med. 2000;343:1445-1453.

38. Romero J, Xue X, Gonzalez W, Garcia MJ. CMR imaging assessing viability in patients with chronic ventricular dysfunction due to coronary artery disease: a metaanalysis of prospective trials. JACC Cardiovasc Imaging. 2012;5:494-508.

39. Bove CM, DiMaria JM, Voros S, Conaway MR, Kramer CM. Dobutamine response and myocardial infarct transmurality: functional improvement after coronary artery bypass grafting--initial experience. Radiology. 2006;240:835-841.

40. Abbas A, Matthews GH, Brown IW, Shambrook JS, Peebles CR, Harden SP. Cardiac MR assessment of microvascular obstruction. Br J Radiol. 2015;88:20140470. doi: 10.1259/ bjr.20140470

41. Wu KC. CMR of microvascular obstruction and hemorrhage in myocardial infarction. J Cardiovasc Magn Reson. 2012;14:68.

42. Anzalone N, Scotti R, Riva R. Neuroradiologic differential diagnosis of cerebral intraparenchymal hemorrhage. Neurol Sci. 2004;25 Suppl 1:S3-S5.

43. Dass S, Suttie JJ, Piechnik SK, Ferreira VM, Holloway CJ, Banerjee R, et al. Myocardial tissue characterization using magnetic resonance noncontrast $\mathrm{T} 1$ mapping in hypertrophic and dilated cardiomyopathy. Circ Cardiovasc Imaging. 2012;5:726-733.

44. Iles LM, Ellims AH, Llewellyn H, Hare JL, Kaye DM, McLean CA, et al. Histological validation of cardiac magnetic resonance analysis of regional and diffuse interstitial myocardial fibrosis. Eur Heart J Cardiovasc Imaging. 2015;16:14-22.

45. McCrohon JA, Moon JC, Prasad SK, McKenna WJ, Lorenz $\mathrm{CH}$, Coats AJ, et al. Differentiation of heart failure related to dilated cardiomyopathy and coronary artery disease using gadolinium-enhanced cardiovascular magnetic resonance. Circulation. 2003;108:54-59.

46. Leyva F, Taylor RJ, Foley PW, Umar F, Mulligan LJ, Patel $\mathrm{K}$, et al. Left ventricular midwall fibrosis as a predictor of mortality and morbidity after cardiac resynchronization therapy in patients with nonischemic cardiomyopathy. J Am Coll Cardiol. 2012;60:1659-1667.

47. Iles L, Pfluger H, Lefkovits L, Butler MJ, Kistler PM, Kaye DM, et al. Myocardial fibrosis predicts appropriate device therapy in patients with implantable cardioverterdefibrillators for primary prevention of sudden cardiac death. J Am Coll Cardiol. 2011;57:821-828.

48. Patel AR, Kramer CM. Role of Cardiac Magnetic Resonance in the Diagnosis and Prognosis of Nonischemic Cardiomyopathy. JACC Cardiovascular Imaging. 2017;10(10 Part A):1180-1193.

49. Semsarian C, Ingles J, Maron MS, Maron BJ. New perspectives on the prevalence of hypertrophic cardiomyopathy. J Am Coll Cardiol. 2015;65:1249-1254.

50. Rickers C, Wilke NM, Jerosch-Herold M, Casey SA, Panse $\mathrm{P}$, Panse $\mathrm{N}$, et al. Utility of cardiac magnetic resonance imaging in the diagnosis of hypertrophic cardiomyopathy. Circulation. 2005;112:855-861. 
51. Anand S, Janardhanan R. Role of cardiac MRI in nonischemic cardiomyopathies. Indian Heart J. 2016;68:405-409.

52. Green JJ, Berger JS, Kramer CM, Salerno M. Prognostic value of late gadolinium enhancement in clinical outcomes for hypertrophic cardiomyopathy. JACC Cardiovasc Imaging. 2012;5:370-377.

53. Mahrholdt H, Goedecke C, Wagner A, Meinhardt G, Athanasiadis A, Vogelsberg $\mathrm{H}$, et al. Cardiovascular magnetic resonance assessment of human myocarditis: a comparison to histology and molecular pathology. Circulation. 2004;109:1250-1258.

54. Ioana G. Lupescu. Amiloidoza cardiaca [Cardiac amyloidosis]. In: SRIM - Radiologie si Imagistica Medicala - Indrumator de studiu pentru pregatirea in specialitate [Medical radiology and imaging - Guide for specialist training] Vol. I. Bucuresti: Ed. Medicala; 2015.

55. Friedrich MG, Sechtem U, Schulz-Menger J, Holmvang G, Alakija P, Cooper LT, et al. Cardiovascular magnetic resonance in myocarditis: A JACC White Paper. J Am Coll Cardiol. 2009;53:1475-1487.

56. Radunski UK, Lund GK, Stehning C, Schnackenburg B, Bohnen S, Adam G, et al. CMR in patients with severe myocarditis: diagnostic value of quantitative tissue markers including extracellular volume imaging. JACC Cardiovasc Imaging. 2014;7:667-675.

57. Lopez-da Costa A, Fernández Armendáriz P, Méndez Diaz C, Rodriguez Garcia E, Díaz Angulo C, Marcos S, Rueda Nuñez F, Soler Fernández R. Magnetic Resonance imaging of pediatric cardiomyopathies. Poster presented at: European Congress of Radiology (ECR); 2016 Mar 2-6; Vienna, AUT.

58. Canter CE, Simpson KE. Diagnosis and treatment of myocarditis in children in the current era. Circulation. 2014; 129:115-128.

59. Hinojar R, Foote L, Arroyo Ucar E, Jackson T, Jabbour $\mathrm{A}, \mathrm{Yu} \mathrm{CY}$, et al. Native $\mathrm{T} 1$ in discrimination of acute and convalescent stages in patients with clinical diagnosis of myocarditis: a proposed diagnostic algorithm using CMR. JACC Cardiovasc Imaging. 2015;8:37-46.

60. Dungu JN, Valencia O, Pinney JH, Gibbs SD, Rowczenio D, Gilbertson JA, et al. CMR-based differentiation of AL and ATTR cardiac amyloidosis. JACC Cardiovasc Imaging. 2014; 7:133-142.

61. Vignaux O. Cardiac sarcoidosis: spectrum of MRI features. AJR Am J Roentgenol. 2005;184:249-254.

62. Greulich S, Deluigi CC, Gloekler S, Wahl A, Zürn C, Kramer $\mathrm{U}$, et al. CMR imaging predicts death and other adverse events in suspected cardiac sarcoidosis. JACC Cardiovasc Imaging. 2013;6:501-511.

63. Manole S. Displazia de ventricul drept (DAVD) [Left ventricle dysplasia]. In: SRIM - Radiologie si Imagistica Medicala - Indrumator de studiu pentru pregatirea in specialitate [Medical radiology and imaging - Guide for specialist training] Vol. II. Bucuresti: Ed. Medicala 2015:324-325.

64. Petersen SE, Selvanayagam JB, Wiesmann F, Robson MD, Francis JM, Anderson RH, et al. Left ventricular non-compaction: insights from cardiovascular magnetic resonance imaging. J Am Coll Cardiol. 2005;46:101-105.

65. Eitel I, von Knobelsdorff-Brenkenhoff F, Bernhardt P, Carbone I, Muellerleile K, Aldrovandi A, et al. Clinical characteristics and cardiovascular magnetic resonance findings in stress (takotsubo) cardiomyopathy. JAMA. 2011;306:277-286.

66. American College of Cardiology Foundation Task Force on Expert Consensus Documents, Hundley WG, Bluemke DA, Finn JP, Flamm SD, Fogel MA, et al. ACCF/ACR/ AHA/NASCI/SCMR 2010 expert consensus document on cardiovascular magnetic resonance: a report of the American College of Cardiology Foundation Task Force on Expert Consensus Documents. Circulation. 2010;55:2614-2662. 